Aim To investigate the association between elective caesarean sections and neonatal respiratory morbidity and the importance of timing of elective caesarean sections.

Methods Cohort study with prospectively collected data of all elective Caesarean sections on mothers with a gestational age of $37+0$ weeks and more, that were performed in our Hospital from 1 January 2011 to 1 January 2012. Multiple pregnancies, fetuses with congenital anomalies, intrauterine deaths, and emergency Caesarean sections were excluded. Primary outcome measures of neonatal respiratory morbidity included transient tachypnoea of newborn, respiratory distress syndrome, persistent pulmonary hypertension of newborn, serious respiratory morbidity (oxygen therapy for more than two days, nasal continuous positive airway pressure).

Results 568 infants were delivered by elective caesarean section. Compared with newborns intended for vaginal delivery, an increased risk of respiratory morbidity was found for infants delivered by elective caesarean section at $37+0$ weeks' gestation to $37+6$ weeks (odds ratio $3.8,95 \%$ confidence interval 2.4 to 6.5 ), $38+0$ weeks' gestation to $38+6$ weeks (3.0, 2.1 to 4.3$)$, and 39+0 weeks' gestation $(1.9,1.2$ to 3.0$)$. The increased risks of serious respiratory morbidity showed the same pattern but with higher odds ratios: a fivefold increase was found at 37 weeks (5.0, 1.6 to 16.0).

Conclusions Infants born by elective caesarean delivery at term are at increased risk for developing respiratory disorders compared with those born by vaginal delivery. A significant reduction in neonatal RDS would be obtained if elective caesarean delivery were performed after $39+0$ gestational weeks of pregnancy.

\section{BRONCHOALVEOLAR LAVAGE IN THE TREATMENT OF MECONIAL ASPIRTION SYNDROME}

doi:10.1136/archdischild-2012-302724.1785

'V Bankovic, 'T Nikolic, 'S Milenkovic, ${ }^{2 B}$ Otasevic. 'Neonatology, Clinical for Gynecology and Obstetrics Clinical Centre of Serbia; ${ }^{2}$ Neonatology, Institute for Neonatology, Belgrade, Serbia

Background and Aims This study was set up to compare two forms of bronchoalveolar lavage (BAL). Lavage with diluted surfactant in saline, and lavage with saline only.

Methods Fifteen term neonates with severe meconial aspiration syndrome (MAS) (needing mechanical ventilation in the first hour and inspiratory oxygen fraction more than $30 \%$ ) were divided into two groups: I group (surfactant group, $\mathrm{n}=8$ ) treated with BAL using diluted surfactant $(6 \mathrm{mg} / \mathrm{ml})$, and II group (saline group, $\mathrm{n}=7$ ), treated with BAL using saline. Both groups were treated with surfactant after BAL.

Results Mean gestational age, Apgar score in the first and the fifth minute, and birth weight did not differ significantly between groups $(p>0.05)$. Duration of mechanical ventilation did not differ between groups (12.5 days vs 17.29; p>0.05), as well as the length of mean length of oxygen therapy (Igroup 6.57 days and 4.57 II group, $p>0.05)$. Also, there was no significant difference in the length of hospital stay (12.5 days vs 17.29; $\mathrm{p}>0.05$ ).

Conclusion Diluted surfactant BAL and saline BAL in the first hours of life combined with a dose of surfactant have the same effect on the length of mechanical ventilation length of oxygen therapy and length of hospital stay.

\section{CHANGE IN EXPRESSION OF PENTRAXIN3-MRNA IN INFECTIOUS FETAL LUNG TISSUES RELATED WITH PREMATURE RUPTURE OF MEMBRANES}

doi:10.1136/archdischild-2012-302724.1786

J Liu, N Yang, XF Wang. Department of Neonatology and NICU, Bayi Children's Hospital Affiliated With General Hospital of Beijing Military Command, Beijing, China
To explore the change in the expression of pentraxin 3 (PTX3)$\mathrm{mRNA}$ and its clinical significance in fetal lung tissues with intrauterine infection related to premature rupture of membranes (PROM).

1. From Oct.2010 to Oct.2011, a total of 12 cases of fetal death, stillbirth, abortion or miscarriage of fetal lung specimens were assigned to this study. Fetal lung samples were devided into two groups according to with or without intrauterine infection related to PROM.

2. Fetal lung tissue specimens were fixed with $4 \mathrm{~g} / \mathrm{L}$ of paraformaldehyde and paraffin embedded within $2 \mathrm{~h}, 4 \mathrm{~mm}$ serial section, and then HE staining. Light microscope was used to identify whether infection/inflammatory response existed in fetal lung tissues or not. Total RNA samples from fresh lung tissure were reverse transcribed into Cdna. Gly ceraldehyde-3-phosphate (GAPDH) was used as reference gene. qRT-PCR and relative quantitative analysis method were performed to detect the expression of mRNA in lung tissue in two groups. Relative expression of Mrna in two groups were compared by $\Delta \mathrm{Ct}$ method of relative quantification.

The results showed that:

1. There was a larger number of neutrophil infiltration, red blood cells leakage, viscous liquid and inflammatory cell exudation in fetal lung tissues of study group.

2. Study group displayed higher expression of PTX3-mRNA than in control group $(5.77 \pm 0.68$ vs. $4.71 \pm 0.40, \mathrm{p}=0.002)$.

Conclusions The expression of PTX3-mRNA was significantly increased in fetal lung tissues with intrauterine infection related with PROM, which can be regarded as a sensitive index for the early diagnosis of fetal intrauterine infection when PROM exists.

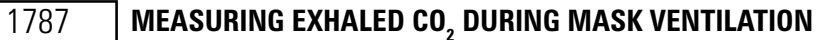 OF PRETERM INFANTS AT BIRTH; A NOVEL TOOL FOR ASSESSING LUNG AERATION?}

doi:10.1136/archdischild-2012-302724.1787

${ }^{1,2,3} \mathrm{G}$ Schmölzer, ${ }^{3,4} \mathrm{O}$ Kamlin, ${ }^{3,4} \mathrm{P}$ Davis, ${ }^{5} \mathrm{~S}$ Hooper. ${ }^{1}$ Department of Pediatrics, University of Alberta, Edmonton, AB, Canada; '2Dept. of Pediatrics, Medical University of Graz, Graz, Austria; ${ }^{3}$ Neonatal Services, The Royal Women's Hospital; ${ }^{4}$ Dept. of Obstetrics \& Gynaecology, The University of Melbourne; ${ }^{5}$ The Ritchie Centre, Monash Institute for Medical Research, Monash University, Melbourne, VIC, Australia

Background Positive pressure ventilation (PPV) remains the cornerstone of respiratory support after birth. Although, gas going in and out of the lung can be measured with a respiratory function monitor, gas exchange (or effective ventilation) may only be determined by presence of exhaled $\mathrm{CO}_{2}$.

Aim To identify non-invasive clinical tools to assess lung aeration in infants receiving PPV after birth.

Methods Deliveries of preterm infants $<32$ weeks gestation were attended. During PPV we measured airway pressures, gas flow and tidal volume $\left(\mathrm{V}_{\mathrm{T}}\right)$, exhaled $\mathrm{CO}_{2}$, heart rate and oxygen saturation. We compared delivered $\mathrm{V}_{\mathrm{T}}$ and heart rate before and after exhaled $\mathrm{CO}_{2}$ was measured.

Results In 10 preterm infants exhaled $\mathrm{CO}_{2}$ and respiratory functions were recorded; their mean (SD) birth weight and gestational age was 27 (2) weeks and 902 (287) grams, respectively. A median (IOR) of 23 (17-43) inflations was delivered with no exhaled $\mathrm{CO}_{2}$. The median (IOR) $\mathrm{V}_{\mathrm{T}}$ when no $\mathrm{CO}_{2}$ was measured was 1.9 (1.0-3.8) $\mathrm{mL} / \mathrm{kg}$ compared to $8.3(2.1-10.3) \mathrm{mL} / \mathrm{kg}$ when exhaled $\mathrm{CO}_{2}$ was measured $(p<0.0025) 1)$. The mean (SD) heart rate while no $\mathrm{CO}_{2}$ was exhaled was 61 (6) beats per minute compared to 104 (41) beats per minute 60 seconds after $\mathrm{CO}_{2}$ was measured.

Conclusion Delivered $\mathrm{V}_{\mathrm{T}}$ and heart rate was significantly lower when no $\mathrm{CO}_{2}$ was exhaled. The presence of exhaled $\mathrm{CO}_{2}$ was accompanied by increases in $\mathrm{HR}$. Combined $\mathrm{V}_{\mathrm{T}}$ measurements, changes in heart rate and detection of exhaled $\mathrm{CO}_{2}$ may provide information on lung aeration of infants transitioning after birth. 